\title{
车轮型面演变对重载固定辙叉磨耗的影响*
}

\author{
朱黄石 ${ }^{1,2}$ 邹小春 ${ }^{1}$ 马贺 ${ }^{1}$ 张 军 $^{1}$ \\ (1. 北京建筑大学城市轨道交通车辆服役性能保障北京市重点实验室 北京 102616 ; \\ 2. 南京地铁运营有限责任公司 南京 210000)
}

\begin{abstract}
摘要: 为研究大秦线路上列车车轮型面演变对固定辙叉动力学性能的影响, 建立重载列车-辙叉动力学模型, 分析重载列车通 过固定辙叉的动力学性能和磨耗规律。结果表明, 车轮通过理论尖端后, 滚动圆半径不断变化使得轮叉接触点线速度发生改 变, 轮叉间滚动与滑动摩擦并存, 对翼轨磨损较大; 不同车轮对心轨冲击位置相对集中, 心轨该区段伤损严重; 车轮演变使 轮叉接触状态发生变化, 磨耗初期车轮的列车动力学性能优于标准车轮, 列车平顺性更好, 磨耗初期机车与货车轮叉垂向力 较标准车轮分别降低了 $39 \%$ 和 $56 \%$, 该磨耗阶段车轮与辙叉匹配时, 辙叉的磨损最轻微。随着车轮型面演变, 车轮磨耗加深, 列车动力学性能逐渐恶化, 磨耗后期车轮对辙叉磨耗掉块伤损的影响最剧烈。
\end{abstract}

关键词: 重载列车; 固定辙叉; 车轮磨耗; 动力学性能; 辙叉伤损

中图分类号: U260

\section{Influence of Wheel Profiles Evolution on Wear of Fixed Frog in Heavy Haul Railway}

\author{
ZHU Huangshi $^{1,2}$ ZOU Xiaochun ${ }^{1}$ MA He $^{1}$ ZHANG Jun ${ }^{1}$ \\ (1. Beijing Key Laboratory of Performance Guarantee on Urban Rail Transit Vehicles, \\ Beijing University of Civil Engineering and Architecture, Beijing 102616;
}

2. Nanjing Metro Operation Co., Ltd., Nanjing 210000)

\begin{abstract}
For Datong-qinhuangdao Line, in order to study the influence of the evolution of the wheel profile on the fixed frog, the dynamic model of heavy haul train and the fixed frog is established to analyze the dynamic performance and wear law of the frog. The results show that after passing the theoretical tip, the radius of the wheel rolling circle changes, causing the linear velocity of the contact point of wheel and frog to change. Besides, for the coexistence of rolling and sliding friction between wheels and frogs, the wing rail wears more seriously. When wheels impact the nose rail, the contact position is relatively concentrated, causing a badly damaged zone on the nose rail. The evolution of the wheel profile changes the contact state between wheels and frogs. Comparing with the standard wheel, the dynamic performance of the worn wheel at the initial stage is better. The ride comfort becomes better and the vertical force becomes lower so that the frog worn slightly. The vertical force of the locomotive wheel and the freight car wheel reduced by $39 \%$ and $56 \%$ respectively. However, with the evolution of the wheel profile, the wear volume increases and the dynamic performance of the wheel becomes worse. At the later stage of wear, the contact friction between the worn wheel and the frog would cause the severe wear and spalling.
\end{abstract}

Key words: heavy haul train; fixed frog; wheel wear; dynamic performance; frog damage

\section{0 前言}

道岔是列车从一股轨道转入另一股轨道的重要 线路连接设备, 是铁路中薄弱环节之一 ${ }^{[1]}$ 。重载线

* 国家自然科学基金(51775031)和北京建筑大学市属高校基本科研业务 费专项资金(X18177)资助项目。20190808 收到初稿, 20191230 收到修 改稿
路上的道岔由于通过列车轴重大等原因，伤损更为 严重。车轮在服役演变过程中型面发生变化, 会影 响与道岔辙叉的接触行为和动态相互作用, 进而影 响辙叉的服役寿命。

对于车轮与道岔的动态相互作用关系, 国内外 专家进行了大量的试验与研究。翟婉明等 ${ }^{[2]}$ 研究了 机车车辆侧向过岔的安全性, 得到侧向极限过岔速 度为 $50 \mathrm{~km} / \mathrm{h}$; 任尊松等 ${ }^{[3-4]}$ 研究了道岔区段轮岔接 
触情况, 给出了两点接触的计算方法和判定条件; 孙宏友等 ${ }^{[5]}$ 评估了动车组与单节货车侧向通过交叉 渡线的安全性情况; BLANCO-SAURA 等 ${ }^{[6]}$ 运用有限 元和多体动力学的方法研究了不同参数对道岔辙叉 区的垂向动态载荷的影响; $\mathrm{KOC}$ 等 ${ }^{[7]}$ 从动力学的角 度, 提出在道岔几何布局的端点, 非线性曲率为 0 最 有利, 对道岔的布局提出了指导。还有学者通过建立 单节车从动力学的角度对道岔结构型面的设计和优 化做了大量研究与创新 ${ }^{[8-10]}$ 。以上研究对列车通过道 岔时动态相互作用的探究以及道岔结构的优化起到 了重要作用, 同时为研究列车通过道岔的动力学性能 和轮叉相互的磨耗影响打下了基础, 但是并未考虑列 车每节车之间的相互作用对列车过叉动力学的影响。

本文基于大秦重载线路现场实测不同磨耗阶段 机车和货车车轮型面, 考虑机车和货车的相互影响, 建立重载列车-辙叉的多体动力学模型, 研究重载列 车车轮型面演变对固定辙叉的动力学性能影响。

\section{1 车轮型面数据测量与分析}

为研究列车车轮型面演变对标准固定辙叉磨耗 的影响, 利用轮轨型面测量仪对大秦重载线路上的机 车和货车车轮型面进行现场跟踪测量, 如图 1 所示。

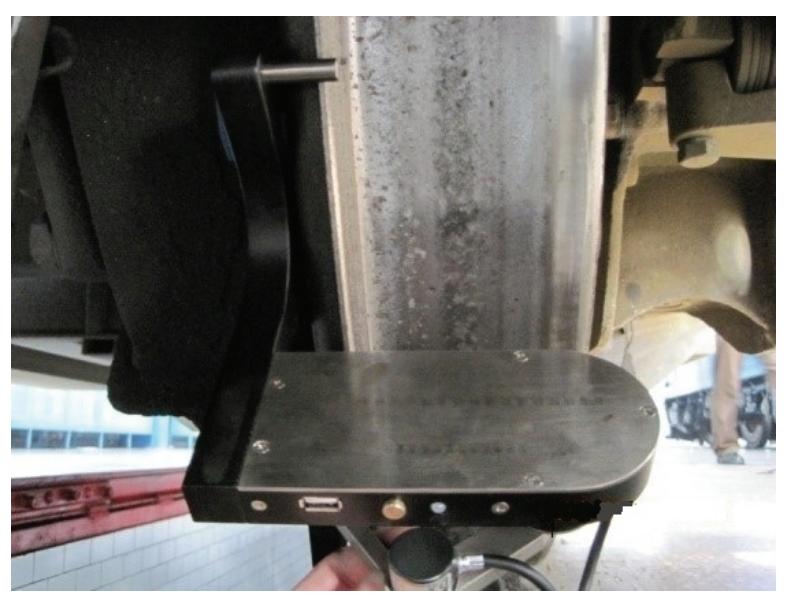

图 1 车轮型面现场测量

大秦重载列车分为机车和货车, 机车车轮标准型 面为 JM3 型, 货车标准型面为 LM 型。对于磨耗后车 轮型面, 可将型面与机车货车的标准型面进行对比, 用车轮型面的垂向磨耗深度来表示车轮的磨耗量, 从 而利用磨耗量的大小来表征车轮的磨耗程度。大秦线 路上车轮的磨耗主要存在踏面中部, 所以选择名义滚 动圆半径附近位置的磨耗量来表示车轮磨耗。

如图 2a 所示为机车车轮型面对比图, 机车标准 车轮型面为 JM3 型。在机车磨耗后车轮型面中按照 磨耗阶段不同选出三条车轮型面, 分别为磨耗初期、
磨耗中期和磨耗后期。如图 $2 \mathrm{~b}$ 表示三种磨耗后车轮 型面名义滚动圆半径附近位置的的磨耗量, 图 2 中 各磨耗后机车车轮磨耗量均呈现出两边低中间高的 趋势, 在 0 20 mm 区域磨耗更为严重。根据磨耗 量将车轮从标准型面至磨耗到限型面分为三个磨耗 阶段如表 1 所示。从三个磨耗阶段中分别选取一个 特征磨耗车轮代表三个磨耗阶段, 机车车轮磨耗初 期的磨耗量约为 $1.2 \mathrm{~mm}$, 磨耗中期的车轮磨耗量约 为 $2.4 \mathrm{~mm}$, 磨耗后期的车轮磨耗量约为 $3.5 \mathrm{~mm}$ 。

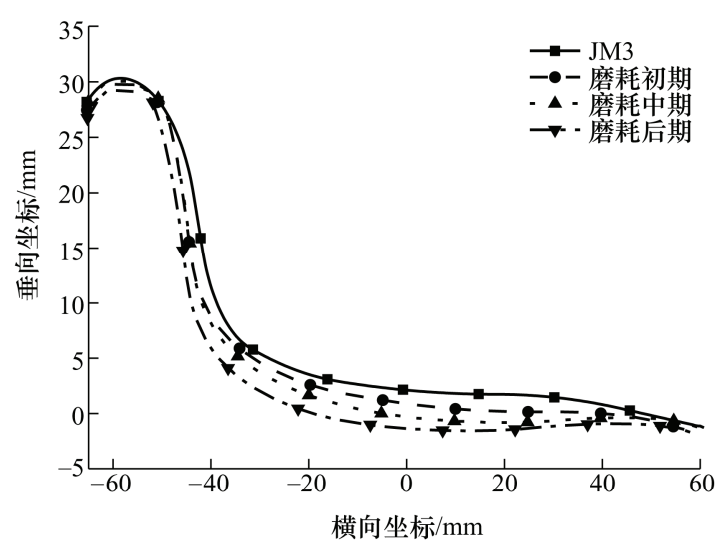

(a) 车轮型面图

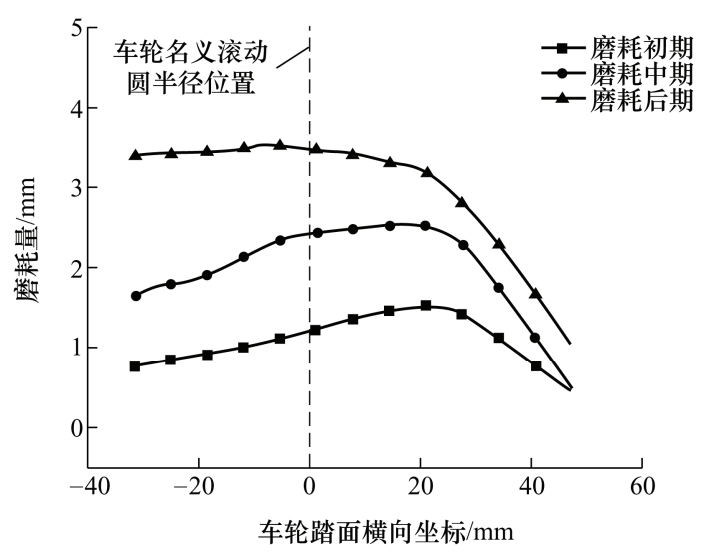

(b) 磨耗后车轮磨耗量

图 2 机车车轮型面及磨耗量

表 1 机车货车磨耗车轮磨耗阶段

\begin{tabular}{ccc}
\hline \multirow{2}{*}{ 磨耗阶段 } & \multicolumn{3}{c}{ 磨耗量 $/ \mathrm{mm}$} \\
\cline { 2 - 3 } & 机车 & 货车 \\
\hline 初期 & $0 \sim 1.6$ & $0 \sim 1.8$ \\
中期 & $1.6 \sim 3.2$ & $1.8 \sim 3.6$ \\
后期 & $3.2 \sim 5.0$ & $3.6 \sim 5.0$ \\
\hline
\end{tabular}

图 3a 为货车车轮各型面对比图, 货车标准车轮 为 $\mathrm{LM}$ 型车轮, 同理选出三条不同磨耗阶段的货车 车轮型面, 三条磨耗后货车车轮型面的磨耗量如图 $3 \mathrm{~b}$ 所示, 货车磨耗后车轮的磨耗量大体趋势与机车 车轮相似, 名义滚动圆半径位置附近磨耗更严重。 同样如表 1 所示将货车磨耗车轮分为三个阶段, 根 
据机车磨耗车轮的定义方法选出三个特征车轮代表 货车车轮磨耗阶段, 货车磨耗初期车轮、磨耗中期 车轮和磨耗后期车轮的魔耗量分别约为 $1.7 \mathrm{~mm}$ 、 $2.9 \mathrm{~mm}$ 和 $4.5 \mathrm{~mm}$ 。

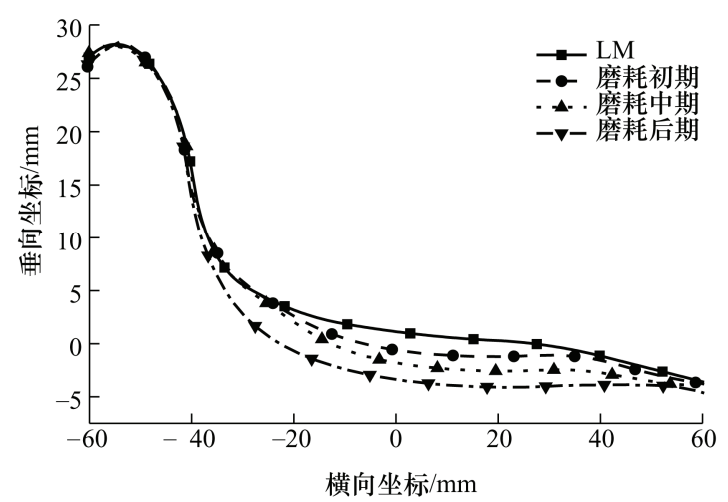

(a) 车轮型面

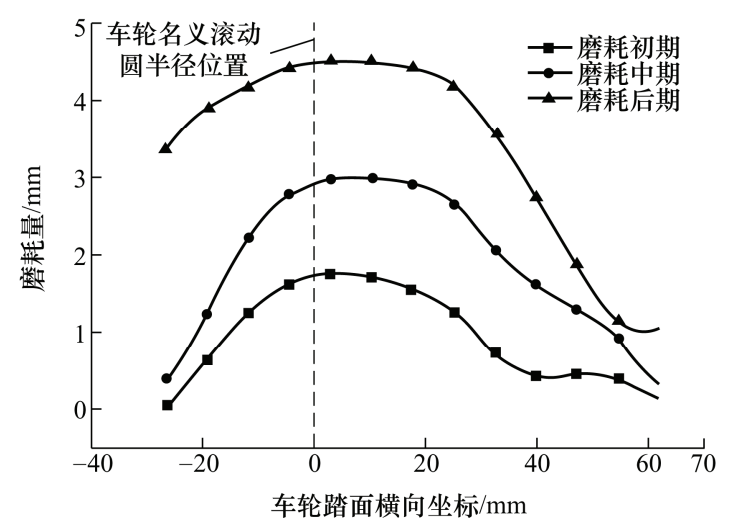

(b) 磨耗后车轮磨耗量

图 3 货车车轮型面与磨耗量

\section{2 计算模型与参数}

\section{1 列车模型的建立}

重载列车的特点之一是车辆节数较多, 需要考 虑列车不同轮位间动力学性能的差异, 根据已完成 的重载列车动力学模型的仿真结果, 第一节货车四个 轮对垂向动力学响应差异较大, 但第二节及以后货车 各轮对垂向动力学响应相近, 故为节约计算成本, 并 体现出首节货车与其他货车的区别, 故本文简化列车 模型为三节车, 第一节为 HXD2 电力机车, 后两节为 C80 货车, 每节车之间用车钩连接。由于本文主要研 究列车过叉时的动力学性能, 在仿真软件中将钩缓装 置简化为非线性弹簧力元, 其中, 力元的刚度为 $2 \mathrm{MN} / \mathrm{m}$, 阻尼为 $40 \mathrm{kN} \cdot \mathrm{s} / \mathrm{m}$ 。列车参数选用大秦重 载线路现场的 $\mathrm{HXD} 2$ 型电力机车和 $\mathrm{C} 80$ 货车参数, 电力机车转向架结构相对简单, 货车采用转 K6 转 向架, 结构更为复杂, 包括摇枕、承载鞍、斜楔和 交叉拉杆等部件。重载列车模型如图 4 所示, 机车
与货车模型部分参数如表 2 所示, 本文选择列车侧向 顺向过叉的限制速度 $50 \mathrm{~km} / \mathrm{h}$ 进行仿真分析。

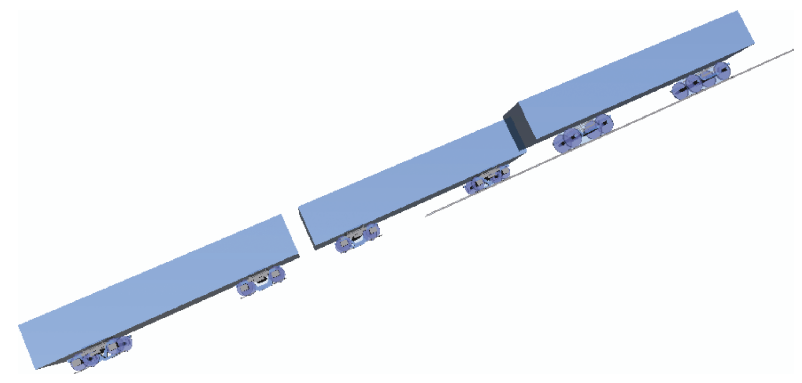

图 4 重载列车模型

表 2 重载列车部分参数

\begin{tabular}{lcc}
\hline \multicolumn{1}{c}{ 参数 } & 机车 & 货车 \\
\hline 名义滚动圆半径 $/ \mathrm{mm}$ & 625 & 420 \\
轴距 $/ \mathrm{m}$ & 2.5 & 1.83 \\
车体质量 $/ \mathrm{kg}$ & 56643 & 24000 \\
构架质量 $/ \mathrm{kg}$ & 12782 & 4108 \\
轮对质量 $/ \mathrm{kg}$ & 1780 & 1200 \\
标准车轮型面类型 & $\mathrm{JM} 3$ & $\mathrm{LM}$ \\
\hline
\end{tabular}

\section{2 辙叉模型建立}

本文采用 $75 \mathrm{~kg} / \mathrm{m}$ 钢轨 12 号固定辙叉, 辙叉各 关键截面如图 5 所示。处理型面时, 首先将辙叉廓 形离散成数据点对来表示, 然后导入软件中将数据 点对拟合成平滑的三次样条曲线, 生成可描述辙叉 截面特征的型面文件。建模过程中, 通过线性插值 的原理自动计算出相邻特征截面间的型面数据, 插 值原理如图 6 所示, 然后通过辙叉控制截面按 Bézier 曲线拉伸成整个辙叉区段。

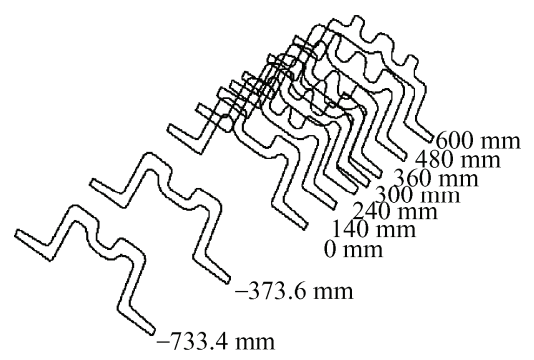<smiles></smiles>

图 5 固定辙叉各关键型面

图 5 中 $0 \mathrm{~mm}$ 位置表示固定辙叉理论尖端, “-”表示该截面位置位于列车顺向过叉时理论尖 端截面前, $-373.6 \mathrm{~mm}$ 截面位置是辙叉咽喉位置, 是两根翼轨最窄处, $-373.6 \sim 0 \mathrm{~mm}$ 截面位置表示 辙叉有害空间区段, 轨道在该区域不连续, 出现了 
轨道间断, 故辙叉该区段是列车易发生事故区域。

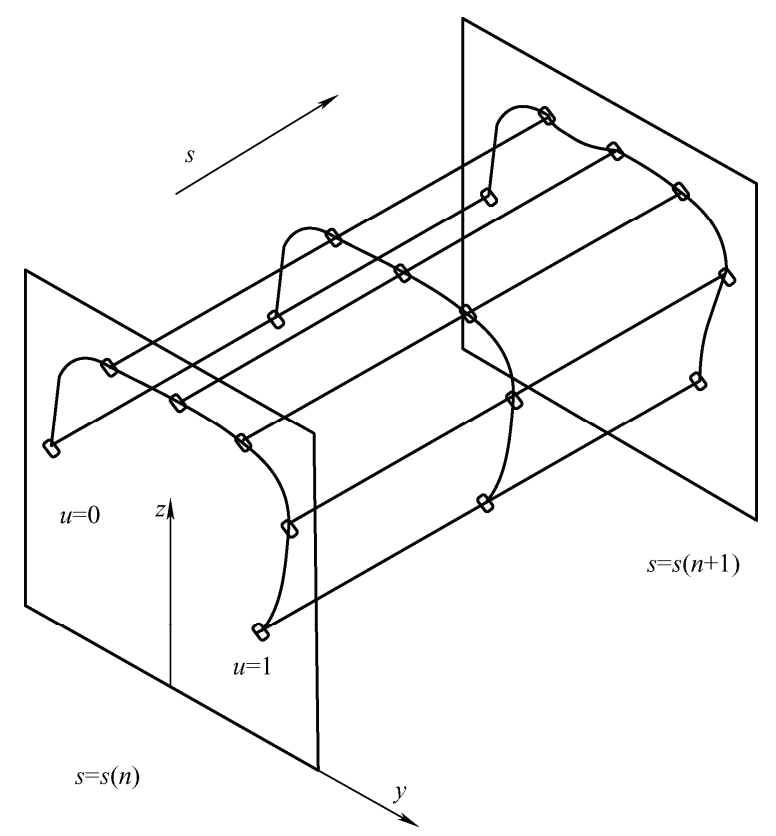

图 6 截面插值原理

辙叉由于其变截面结构的特点, 各型面位置的 宽度和高度均随纵向不断变化, 故即使在标准车轮 通过标准固定辙叉的情况下, 轮叉接触位置依然发 生横向和垂向的变化, 这种变化是由于辙叉结构特 点所造成的固有不平顺, 故称为辙叉的 “结构不平 顺” [11]。辙叉结构不平顺是引起列车过叉激振的主 要原因之一。

\section{3 计算结果分析}

\section{1 滚动圆半径}

选取辙叉的关键区域, 即理论尖端前后 $2 \mathrm{~m}$ 的 区段作为研究对象, 探究列车车轮通过辙叉时轮叉 接触点的滚动圆半径变化规律。通过分析可知, 轮 叉接触点滚动圆半径的变化规律主要与车轮型面相 关, 故仅对机车货车的首轮对进行研究, 得到轮叉 接触点的滚动圆半径规律如图 7 所示。对比机车与 货车, 可知机车货车车轮过叉时接触点滚动圆半径 变化规律相似, 车轮顺向过叉通过理论尖端后, 轮 叉接触点相比于轮缘位置逐渐外移, 故接触点的滚 动圆半径逐渐减小。当车轮从翼轨过渡到心轨时, 轮叉接触点从远离轮缘一侧直接突变到踏面中部区 域, 相对应滚动圆半径的曲线也存在突变剧增, 过 渡完成后, 接触点滚动圆半径逐步趋于平稳, 回归 到初始值附近。

机车和货车标准车轮的名义滚动圆半径分别为 $625 \mathrm{~mm}$ 和 $425 \mathrm{~mm}$ 。从图 $7 \mathrm{a}$ 中可知, 机车车轮接
触点滚动圆半径在理论尖端后 $0.43 \sim 0.48 \mathrm{~m}$ 区段发 生突变, 突变值为 $4.2 \mathrm{~mm}$ 。图 $7 \mathrm{~b}$ 中可知, 货车车 轮接触点滚动圆半径突变位置在理论尖端后 $0.41 \sim$ $0.45 \mathrm{~m}$ 区段, 相较机车车轮过渡位置更靠前, 突变 值为 $5.8 \mathrm{~mm}$ 。根据以上分析可知, 滚动圆半径突变 的位置即机车与货车车轮从翼轨向心轨过渡区段。

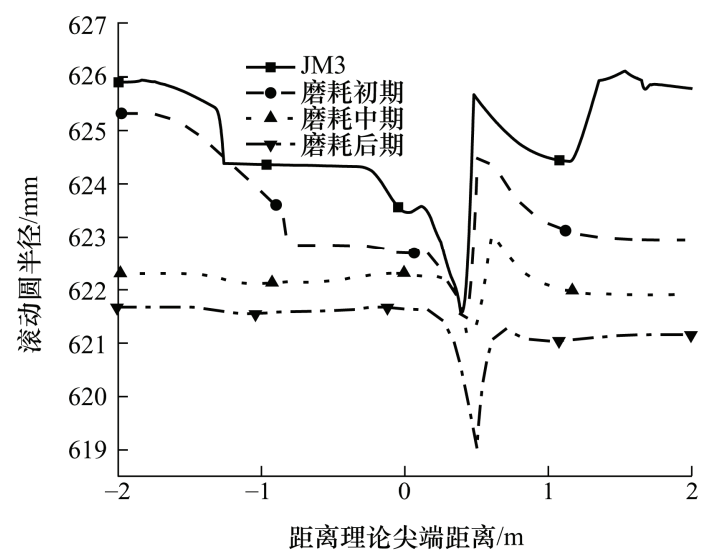

(a) 机车车轮滚动圆半径

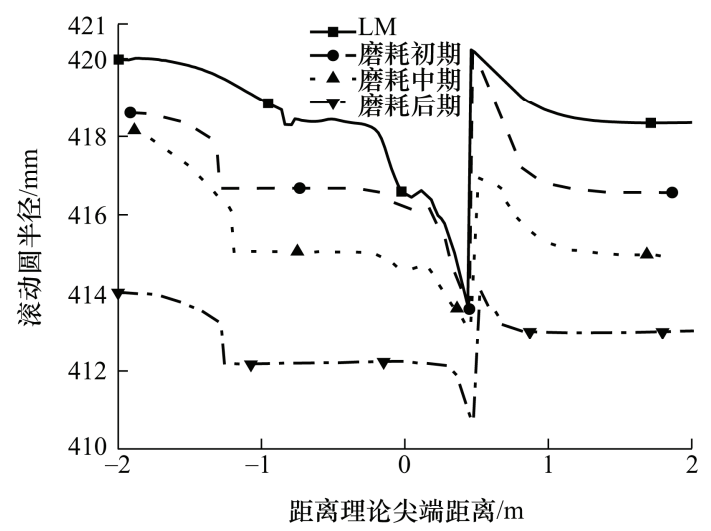

(b) 货车车轮滚动圆半径

图 7 列车车轮滚动圆半径

现场测量的机车和货车磨耗后车轮滚动圆半径 突变位置与标准车轮相差不大, 但由于磨耗后车轮 踏面中部磨耗严重, 改变了车轮踏面雉度, 故磨耗 后机车货车车轮滚动圆半径突变量较标准车轮更 小, 机车三种磨耗后车轮滚动圆半径突变值最小仅 为 $2.4 \mathrm{~mm}$, 货车三种磨耗后车轮滚动圆半径突变值 最小仅为 $3.4 \mathrm{~mm}$ 。

在列车顺向过叉驶过理论尖端后, 列车整体速 度保持恒定, 但由于轮叉接触点滚动圆半径的减小 而导致车轮线速度降低。车轮与翼轨滚动接触与滑 动接触共存, 因此产生了较大的纵向蠕滑力, 最大 可达到 $61 \mathrm{kN}$, 与翼轨磨损剧烈。当车轮从翼轨向 心轨过渡前的瞬间, 滚动圆半径降到最小, 左右轮 径差达到最大, 此时车轮与翼轨滑动摩擦最大, 造 成该处磨损最严重, 与现场磨耗情况一致。 


\section{2 轮叉垂向力}

货车节数多是重载列车的特征之一，货车不同 节车不同轮位间动力学性能均存在差异, 故以货车 标准车轮为研究对象, 研究不同轮位的动力学性能 的差异。根据已完成的动力学仿真结果显示, 每节 货车的首轮对, 因为最早受到冲击, 轮叉垂向力相 较于同节车其他轮对更大，故选取不同节货车的第 一轮对比较其轮叉垂向力的差异。

同样选取理论尖端前后 $2 \mathrm{~m}$ 区段进行研究, 货 车第一节车第一轮对和第二节车第一轮对垂向力变 化如图 8 所示。图 8 中可看出, 两组轮叉垂向力的 变化趋势基本相同, 比较具体数值, 第一节货车第 一轮对的轮叉垂向力更大。两组轮轮叉垂向力在 车轮从翼轨向心轨的过渡位置均达到最大值, 对比 数值, 第一节货车第一轮对和第二节货车第一轮对 的最大垂向力分别为 $518 \mathrm{kN}$ 和 $414 \mathrm{kN}$, 第一节货 车第一轮对的轮叉垂向力最大值较第二节货车第一 轮对增大了约 $25 \%$, 第一节货车动力学响应更剧 烈。因此, 对货车垂向力的分析中, 选取第一节货 车第一轮对作为研究对象。

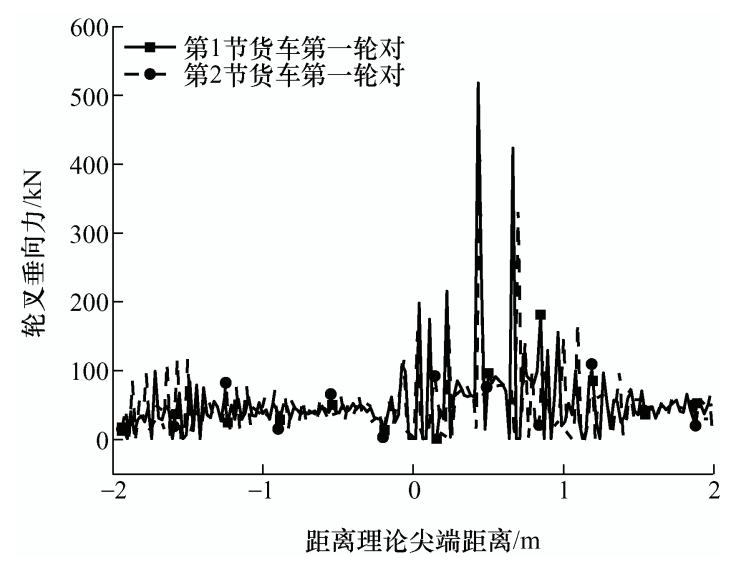

图 8 货车不同轮对垂向力

机车车轮第一轮对的轮叉垂向力变化如图 9a 所 示，机车不同类型轮对的轮叉垂向力变化规律基本相 同, 当机车车轮驶过理论尖端后, 轮对轮叉垂向力开 始波动加剧。机车标准和磨耗后车轮垂向力最大值集 中在理论尖端后 $0.45 \sim 0.55 \mathrm{~m}$ 区域, 根据图 9a 中显 示, 磨耗后车轮垂向力最大值出现的位置较标准车轮 后移, 且随着磨耗程度加深最大值位置更靠后。结合 第 3.1 节滚动圆半径变化分析可知, 该区段是车轮从 翼轨向心轨过渡位置, 易对心轨造成强烈冲击。

货车车轮垂向力的变化如图 9b 所示, 选择第一 节货车第一轮对作为研究对象。从图 $9 b$ 中可看出, 货车车轮轮叉垂向力的变化规律大致与机车车轮相 同, 顺向过叉时, 车轮在通过理论尖端后垂向力波
动加剧, 且垂向力最大值出现的区域较机车车轮更 加集中，主要在距离理论尖端 $0.44 \sim 0.52 \mathrm{~m}$ 区域, 不同磨耗车轮冲击心轨位置相差不大, 同样货车各 车轮的轮叉垂向力峰值均处于车轮从翼轨向心轨过 渡区段。

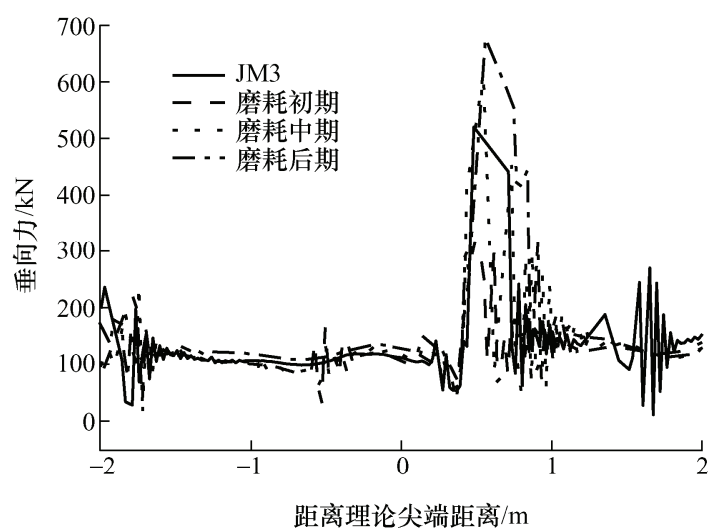

(a) 机车轮叉垂向力

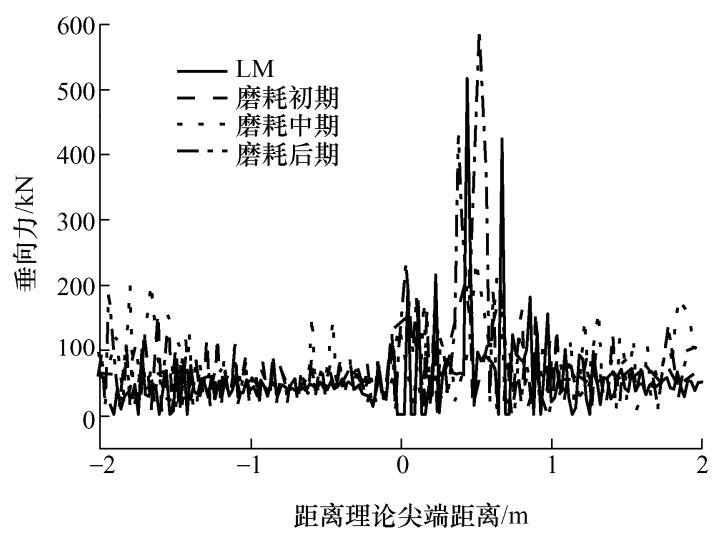

(b) 货车轮叉垂向力

图 9 列车轮叉垂向力

不同类型车轮轮叉垂向力最大值如图 10 所示。 机车标准车轮和货车标准车轮最大轮叉垂向力大致 相同, 分别为 $522 \mathrm{kN}$ 和 $518 \mathrm{kN}$ 。随着列车现场服 役, 车轮开始磨耗, 当车轮达到磨耗初期阶段时, 列车轮叉垂向力大幅度减小, 机车与货车最大轮叉 垂向力仅为 $320 \mathrm{kN}$ 和 $226 \mathrm{kN}$, 相较于标准车轮分 别减少 $39 \%$ 和 $56 \%$ 。随着车轮磨耗增加, 机车磨耗 中期与货车磨耗后期车轮轮叉垂向力陡增, 超过标 准车轮与辙叉的最大垂向力。对于磨耗后车轮, 机 车车轮最大垂向力逐渐大于货车车轮最大垂向力, 对辙叉心轨垂向冲击更大。

综上所述, 机车与货车各车轮最大垂向力位置 较为集中, 主要分布在理论尖端后 $0.44 \sim 0.55 \mathrm{~m}$ 心 轨上, 该区段为辙叉心轨上磨耗掉块损伤最严重区 段, 与现场调研情况一致, 模型与仿真结果可靠。 随着列车车轮型面演变, 轮叉垂向力呈现先减小后 增大的趋势, 磨耗后期车轮对辙叉的损伤更大。 


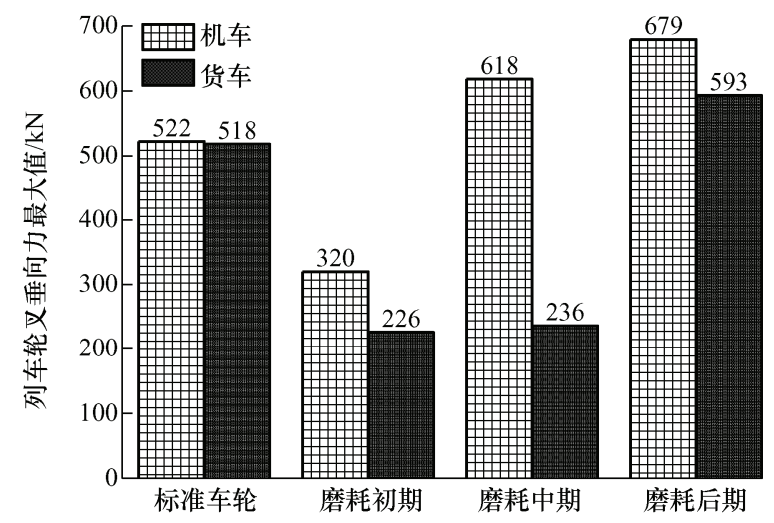

图 10 列车车轮最大垂向力

\section{3 平顺性}

应用机车和货车第一轮对垂向位移的波动来直 接表示列车过叉平顺性 ${ }^{[12]}$ 。

列车顺向通过固定辙叉时, 首轮对轮轴轴心垂 向波动情况如图 11 所示, 图中正值表示车轮轴心向 上波动。对比图 11 两张图可看出, 机车货车过叉时 轴心垂向位移波动大致趋势相同, 车轮垂向位置在 理论尖端前波动较小, 靠近理论尖端时, 轮轴垂心 垂向位置逐渐降低, 从前文辙叉各关键截面型面可 看出不同位置辙叉高度发生变化, 再加上轮叉接触 点的位置不断远离轮缘, 故车轮轴心垂向位置逐渐 降低。车轮随后从翼轨向心轨过渡, 随着心轨的抬 高, 垂向位置出现上升的趋势, 完全过渡后, 趋于 平稳。车轮在该区域垂向位移的变化与图 7 中所示 滚动圆半径的变化规律相近。

机车标准车轮过叉时垂向位移整体变化在 $1 \mathrm{~mm}$ 内, 机车磨耗初期车轮平顺性与标准车轮差 别较小, 甚至更优于机车标准车轮, 而磨耗中期车 轮垂向位移在理论尖端后波动增加, 车轮轴心最大 波动达 $1.7 \mathrm{~mm}$, 磨耗后期车轮波动更大, 机车从翼 轨向心轨过渡时, 车轮轴心垂向波动达 $2.3 \mathrm{~mm}$, 该 磨耗阶段机车车轮过叉时的平顺性更差。

货车过叉时轮对垂向波动较机车更小。标准 车轮、磨耗初期和磨耗中期车轮平顺性较好, 波 动均保持在 $0.7 \mathrm{~mm}$ 内, 随着车轮磨耗, 磨耗后 期货车车轮从翼轨向心轨过渡时波动增大, 达到

\section{$1.4 \mathrm{~mm}$ 。}

综上对比分析, 列车磨耗初期车轮通过固定辙 叉时, 平顺性较标准车轮更优, 磨耗后期车轮平顺 性较差, 且货车过叉平顺性优于机车。较差的平顺 性意味着车轮垂向波动更大, 导致车轮对辙叉垂向 冲击更严重, 在服役过程中, 货车车轮垂向波动更 轻微, 结合垂向力结果分析, 货车轮叉垂向力也更 小, 与上文垂向力得到结论一致。

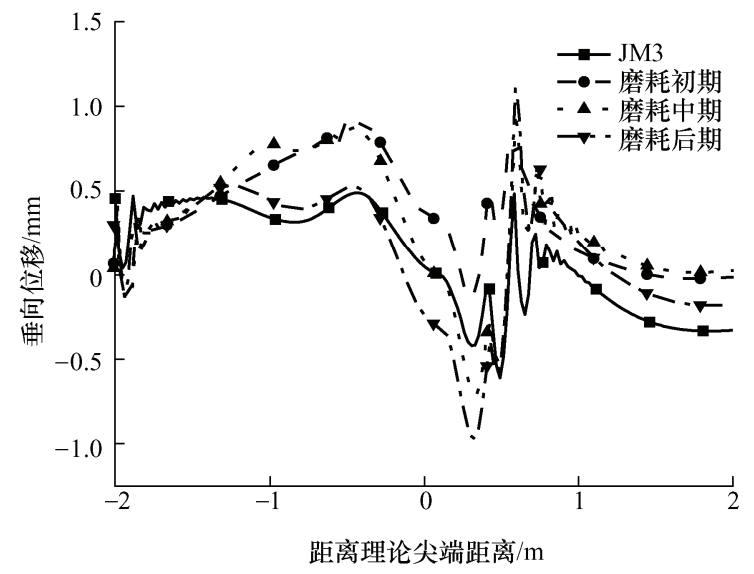

(a) 机车过叉平顺性

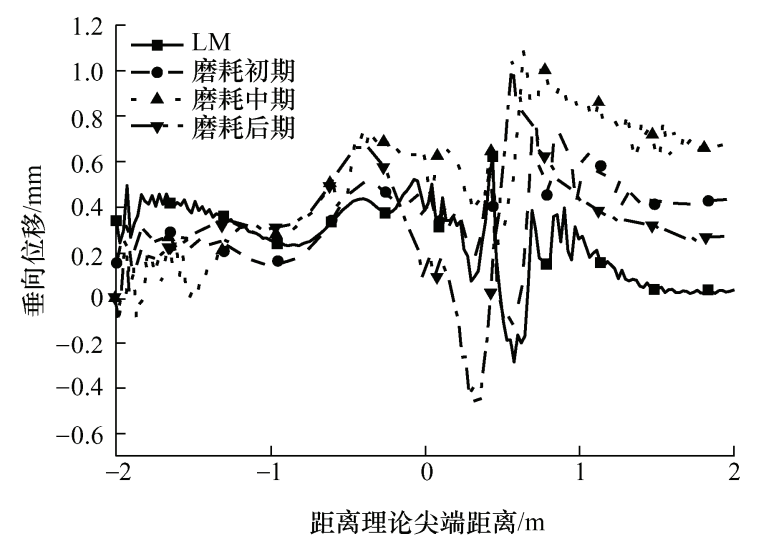

(b) 货车过叉平顺性

图 11 列车过叉平顺性

\section{4 磨耗数}

磨耗数通常用接触斑内的蠕滑力乘以蠕滑率来 表示, 可一定程度上表示轮叉的磨耗程度, 磨耗数 越大表示磨耗程度越深 ${ }^{[13]}$

$$
W=T_{\mathrm{x}} \xi_{\mathrm{x}}+T_{\mathrm{y}} \xi_{\mathrm{y}}
$$

式中, $T_{\mathrm{x}}$ 为横向蠕滑力; $T_{\mathrm{y}}$ 为纵向蠕滑力; $\xi_{\mathrm{x}}$ 为横 向蠕滑率; $\xi_{\mathrm{y}}$ 为纵向蠕滑率。

机车与货车车轮通过固定辙叉的过程中磨耗数 的变化如图 12 所示。图 12a 中可看出, 机车车轮通 过理论尖端前, 磨耗数波动不大, 通过理论尖端后, 磨耗数开始波动加剧, 当车轮从翼轨向心轨过渡前 后, 磨耗数陡增并达到峰值。结合第 3.1 节滚动圆 半径分析, 车轮在该区段, 滚动圆半径变化导致轮 对出现轮径差, 从而纵向蠕滑率增加, 轮叉相互磨 耗较严重。磨耗后期机车车轮磨耗数峰值远超过其 他型面车轮, 达到 $2081 \mathrm{~N}$, 与辙叉磨损剧烈。而 磨耗初期机车车轮磨耗数则更平稳, 峰值较小, 该 磨耗阶段机车车轮对翼轨和心轨损伤相对较小。

图 12b 则表示货车顺向过叉时磨耗数的变化情 况。相较于机车车轮, 总体上货车车轮的磨耗数小 于机车, 货车磨耗数峰值同样出现在车轮从翼轨向 
心轨过渡区段, 该区段翼轨和心轨损伤严重。货车 标准车轮和磨耗后期车轮磨耗数峰值较大, 分别达 到 $975 \mathrm{~N}$ 和 $1538 \mathrm{~N}$, 故货车该两种型面车轮与标 准辙叉磨耗会更加剧烈。磨耗初期车轮磨耗数较为 平稳, 处于稳定磨耗阶段, 与辙叉磨损最小 ${ }^{[14]}$ 。

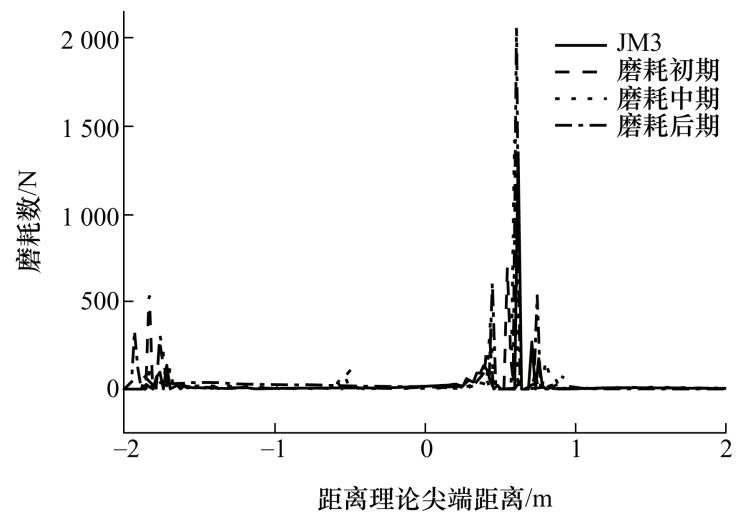

(a) 机车磨耗数

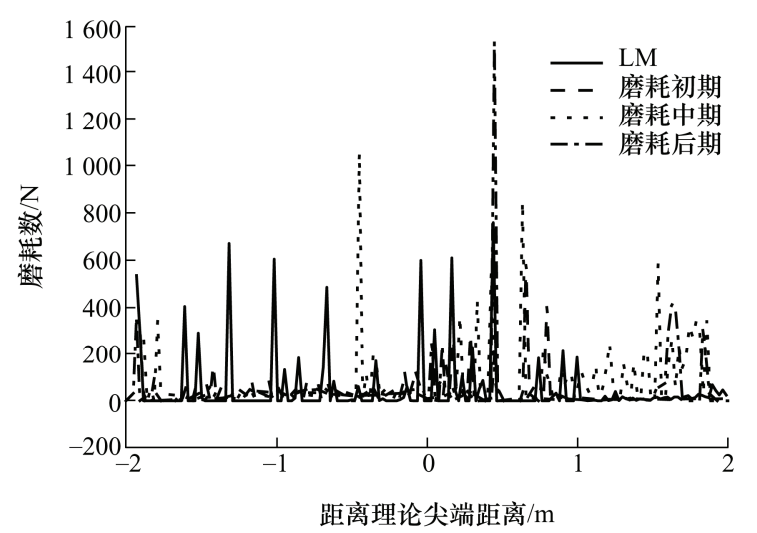

(b) 货车磨耗数

图 12 列车磨耗数

综上可知, 列车通过固定辙叉时, 机车车轮与 辙叉间的磨耗数大于货车车轮, 机车对辙叉的伤损更 大, 且磨损主要集中于车轮从翼轨向心轨过渡区段。

\section{4 结论}

本文通过建立重载列车-辙叉模型, 探究车轮型 面演变对重载列车通过固定辙叉动力学性能的影 响, 得到以下结论。

(1) 列车车轮通过理论尖端后, 滚动圆半径逐 渐减小, 车轮线速度降低, 导致车轮与翼轨接触时 滚动摩擦和滑动摩擦同时存在, 纵向蠕滑力增大, 对翼轨表面磨损较大, 这是辙叉翼轨磨损的重要原 因之一。

(2) 磨耗初期车轮的列车平顺性更优, 故相较 于标准车轮, 磨耗初期车轮大幅度降低了轮叉间垂 向力, 对辙叉心轨损伤更小, 而磨耗后期车轮时列 车平顺性恶化, 对心轨冲击最大。列车各车轮的轮
叉垂向力峰值均集中在理论尖端后 $0.44 \sim 0.55 \mathrm{~m}$ 心 轨上的区段, 该处易发生磨耗掉块伤损，与现场情 况一致。

(3) 相较于货车，机车与固定辙叉间磨耗数更 大, 列车磨耗初期车轮比标准车轮磨耗数更小, 标 准和磨耗后期车轮磨耗数均较大, 会加剧轮叉相互 作用, 缩短辙叉寿命。

综上可知, 随着列车车轮演变, 列车动力学性 能呈现先变优再恶化的趋势, 磨耗初期车轮与辙叉 匹配时, 列车动力学性能最优, 辙叉的磨耗伤损最 轻微。

\section{参 考 文 献}

[1] 罗望, 吴安伟. 动车组与单节车侧向通过道岔动力学性 能比较 $[\mathrm{J}]$. 机车电传动, 2007(1): 5-7.

LUO Yun, WU Anwei. Comparison between dynamic performances of EMUs and single locomotive when passing a turnout[J]. Electric Drive for Locomotives, 2007(1): 5-7.

[2] 翟婉明, 王开云. 机车车辆侧向通过道岔时的运行安全 性评估[J]. 同济大学学报, 2004, 32(3): 382-386.

ZHAI Wanming, Wang Kaiyun. Safety assessment of trains passing through branch lines of turnouts[J]. Journal of Tongji University, 2004, 32(3): 382-386.

[3] 任尊松, 孙守光. 道岔区轮轨接触几何关系研究 [J]. 工 程力学, 2008(11): 223-230.

REN Zunsong, SUN Shouguang. Study on the wheel / rail, contact geometry relation of the turnout zone[J]. Engineering Mechanics, 2008，25(11): 223-230.

[4] 任尊松, 翟婉明, 王其昌. 轮轨接触几何关系在道岔系 统动力学中的应用[J]. 铁道学报, 2001(5): 11-15.

REN Zhunsong, ZHAI Wanming, WANG Qichang. The use of spatial wheel/rail contact geometric relationship in the turnout system dynamics[J]. Journal of the China Railway Society, 2001(5): 11-15.

[5] 孙宏友, 王平, 张东风, 等. 动车组与货车侧向通过整 体道床 12 号交叉渡线道岔动力学特性分析 [J]. 铁道标 准设计, 2015，59(5): 70-73.

SUN Hongyou, WANG Ping, ZHANG Dongfeng, et al. Dynamics analysis of EMUS and freight car passing through No.12 crossover turnout with solid bed[J]. Railway Standard Design, 2015，59(5): 70-73.

[6] BLANCO-SAURA A E, VELARTE-GONZALEZ J L, RIBES-LLARIO F, et al. Study of the dynamic vehicletrack interaction in a railway turnout[J]. Multibody System Dynamics, 2017(1): 1-16. 
[7] KOC W, PALIKOWAKA K. Dynamic analysis of the turnout diverging track for HSR with variable curvature sections[J]. World Journal of Engineering \& Technology, 2017, 5(1): 42-57.

[8] 曹洋, 王平, 杨生. 道岔平面选型的动力学研究 $[J]$. 华 中科技大学学报, 2017, 45(11): 35-40.

CAO Yang, WANG Ping, YANG Sheng. Dynamic study on turnout plane alignment selection[J]. Journal of Huazhong University of Science and Technology, 2017, 45(11): 35-40.

[9] 陈漫, 王平. 钢轨廓形优化对转辙器动力特性的影响研 究[J]. 铁道标准设计, 2017, 61(10): 37-42, 60 .

CHEN Man, WANG Ping. Research on impact of rail profile optimization on dynamic characteristics of switch[J]. Railway Standard Design, 2017，61(10): 37-42， 60.

[10] 王树国, 司道林, 杨东升, 等. 我国高速铁路道岔尖轨 廓形研究[J]. 中国铁路, 2018(1): 15-19.

WANG Shuguo, SI Daolin, YANG Dongsheng, et al. Switch rail profiles of HSR in China[J]. China Railway, 2018(1): 15-19.

[11] 赵卫华. 固定辙叉轮轨关系优化及动力学仿真分析研 究[D]. 成都: 西南交通大学, 2014 .

ZHAO Weihua. Study on wheel/rail contact geometry optimization and dynamic simulation for rigid frog[D]. Chengdu: Southwest Jiaotong University, 2014.

[12] 李俊楠. 机车通过重载固定辙叉系统动力学研究[D].大 连：大连交通大学，2015.

LI Junnan. Dynamic study on the locomotive wheel and rigid frog of heavy rail[D]. Dalian: Dalian Jiaotong University, 2015.

[13] 陆文教, 陶功权, 王鹏, 等. 地铁车轮磨耗对轮轨接触特 性及动力学性能的影响[J]. 工程力学, 2017, 34(8): 222-231.

LU Wenjiao, TAO Gongquan, WANG Peng, et al. Influence of wheel wear on wheel-rail contact behavior and dynamic performance of metro vehicle $[\mathrm{J}]$ Engineering Mechanics, 2017, 34(8): 222-231.

[14] 朱黄石. 重载列车对固定辙叉的磨耗及动力学性能研 究[D]. 北京: 北京建筑大学, 2019 .

ZHU Huangshi. Study on wear and dynamics performance of heavy-duty trains on fixed frogs[D]. Beijing: Beijing University of Civil Engineering and Architecture, 2019.

作者简介: 朱黄石, 男, 1995 年出生。主要研究方向为轮轨关系。 E-mail:530434429@qq.com

马贺(通信作者), 女, 1988 年出生, 博士, 讲师。主要研究方向为轮轨 关系。

E-mail: 13120171199@163.com 